\title{
Chernoff Upper Bound of PEP for Distributed Space-Time Coding Relay Systems in Nakagami- $m$ Fading Channels
}

\author{
Nan Wang ${ }^{1}$, Ming Chen ${ }^{1}$, Wence Zhang ${ }^{1}$, Xiaoli $\mathrm{Chu}^{2}$ \\ ${ }^{1}$ National Mobile Communications Research Laboratory, Southeast University, 210096, Nanjing, P. R. China \\ ${ }^{2}$ Division of Engineering, King's College London, London WC2R 2LS, UK \\ \{wangnan,chenming,wencezhang\}@seu.edu.cn, xiaoli.chu@kcl.ac.uk
}

\begin{abstract}
The Chernoff upper bound of the pairwise error probability (PEP) and a closed-form approximation for distributed space-time coding (DSTC) relay systems in Nakagami- $m$ fading channels are derived in this paper, numerical results show that the upper bound approximation is quite near to the real value of the Chernoff upper bound of the PEP.
\end{abstract}

Keywords- distributed space-time coding relay system; Nakagami-m fading; pairwise error probability;approximation of upper bound

\section{INTRODUCTION}

Applying relay to assist the signal transmission of wireless communications system can combat the channel fading and increase the capacity, performance and coverage. So it has been widely investigated, and many schemes of applying relay have been proposed. One method to apply relay is to carry out distributed space-time coding (DSTC) between a group of relays [1]-[4], and it has been shown that it is an effective approach to improve the spectrum efficiency. In a DSTC-relay system, the transmitter sends the signals to the relays in the first phase and the relays carry out a linear transformation which is named DSTC to the received signals, and then send the coded signals to the receiver in the second phase.

Although there have been many work on performance analysis of various DSTC-relay systems, most of them were given under the assumption that the channel fading is Rayleigh or Rician [2], [5]. The performance of DSTC-relaying system under more generalized Nakagami- $m$ fading channel has not been investigated, to the best of our knowledge.

In this paper, we derive the closed-form expression approximation of the Chernoff bound of the pairwise error probability (PEP) for an Alamouti DSTC-relay system in nonidentical Nakagami- $m$ fading channels. Numerical results are also given to show the validity of the approximation.

Bold capitalized letters denote matrices in the paper, bold lowercase letters denote vectors. For a matrix $\boldsymbol{A}, \boldsymbol{A}^{\mathrm{T}}, \overline{\boldsymbol{A}}$,
$\boldsymbol{A}^{\mathrm{H}}$, and $\|\boldsymbol{A}\|$ denote the transpose, conjugate, Hermitian, and Frobenius norm of $\boldsymbol{A}$ respectively.

\section{SYSTEM MODEL}

Consider a relay system with a transmitter, a receiver, and two relays shown in Fig. 1. Every node in the system has only a single antenna.



Figure 1 A relay system with two relays

In the first phase, the two relays receive the two-time-slots signals $s_{1}$ and $s_{2}$ from the transmitter, let $s=\left(s_{1}, s_{2}\right)^{\mathrm{T}}$, and they satisfy $E\left\{s^{\mathrm{H}} s\right\}=1$. And in the second phase, the two relays code the received two-time-slots signals and transmit the coded signals to the receiver in another two time slots.

The wireless channels in each phase are assumed to keep unchanged, the channel vectors of the two phases are denoted respectively by $\boldsymbol{f} \triangleq\left(f_{1}, f_{2}\right)^{\mathrm{T}}$ and $\boldsymbol{g} \triangleq\left(g_{1}, g_{2}\right)^{\mathrm{T}}$, all the channel coefficients are assumed to be independent and of Nakagami- $m$ distribution, the PDF of $f_{1}, f_{2}$ and $g_{1}, g_{2}$ are respectively assumed to be

$$
f(x)=\frac{2 m^{m} x^{2 m-1}}{\Omega^{m} \Gamma(m)} \exp \left(-\frac{m x^{2}}{\Omega}\right)
$$

and

$$
f(y)=\frac{2 n^{n} y^{2 n-1}}{\omega^{n} \Gamma(n)} \exp \left(-\frac{n y^{2}}{\omega}\right)
$$

where $m, n$ and $\Omega, \omega$ are respectively severity fading parameters and power parameters of the channels in the two phases. 
Denote the two-time-slots received signals and noises at relay $i(i=1,2)$ respectively by $\boldsymbol{r}_{i}$ and $\boldsymbol{v}_{i}$ with following expressions

$$
\boldsymbol{r}_{i}=\left[\begin{array}{c}
r_{i, 1} \\
r_{i, 2}
\end{array}\right], \quad \boldsymbol{v}_{i}=\left[\begin{array}{c}
v_{i, 1} \\
v_{i, 2}
\end{array}\right]
$$

Suppose that the total power of the transmitter is $2 P$, then we have

$$
\boldsymbol{r}_{i}=\sqrt{2 P} f_{i} \boldsymbol{s}+\boldsymbol{v}_{i}
$$

where the noises are assumed to be i.i.d. variables with distribution $\mathcal{C N}(0,1)$.

In the second phase, the relays code their received two-timeslots signals using the following Alamouti DSTC:

$$
\boldsymbol{t}_{1}=\frac{\sqrt{0.5 P}}{\sqrt{\Omega P+1}} \boldsymbol{r}_{1} \quad \boldsymbol{t}_{2}=\frac{\sqrt{0.5 P}}{\sqrt{\Omega P+1}}\left(\begin{array}{cc}
0 & -1 \\
1 & 0
\end{array}\right) \overline{\boldsymbol{r}}_{2}
$$

The coefficient $\sqrt{0.5 P} / \sqrt{\Omega P+1}$ is to ensure that the average transmit power for one transmission at every relay is $0.5 P$, that is , $\mathrm{E}\left\{\boldsymbol{t}_{i}^{*} \boldsymbol{t}_{i}\right\}=0.5 P \times 2$. Denote the received signal and noise at the receiver by $\boldsymbol{x}$ and $\boldsymbol{w}$ with following expressions

$$
\boldsymbol{x}=\left[\begin{array}{l}
x_{1} \\
x_{2}
\end{array}\right], \quad \boldsymbol{w}=\left[\begin{array}{l}
w_{1} \\
w_{2}
\end{array}\right]
$$

$w_{1}, w_{2}$ are i.i.d variables with the distribution $\mathcal{C N}(0,1)$. After some simple computation, $\boldsymbol{x}$ can come to the following expression

$$
\begin{aligned}
\boldsymbol{x}=\sqrt{2 P} & \left(\begin{array}{cc}
s_{1} & -\bar{s}_{2} \\
s_{2} & \bar{s}_{1}
\end{array}\right)\left[\begin{array}{l}
\sqrt{0.5 P} f_{1} g_{1} / \sqrt{\Omega P+1} \\
\sqrt{0.5 P} f_{2} g_{2} / \sqrt{\Omega P+1}
\end{array}\right] \\
+ & \frac{\sqrt{0.5 P}}{\sqrt{\Omega P+1}} g_{1} \boldsymbol{v}_{1}+\frac{\sqrt{0.5 P}}{\sqrt{\Omega P+1}} g_{2}\left(\begin{array}{cc}
0 & -1 \\
1 & 0
\end{array}\right) \overline{\boldsymbol{v}}_{2}+\boldsymbol{w}
\end{aligned}
$$

\section{THE UPPER BOUND ESTIMATION OF PEP}

The PEP is known to be a good performance measure for a block-fading system. In this section, we consider to give a closed-form expression of the Chernoff upper bound on the PEP, which is a tight upper bound on the error rate [5].

The maximum-likelihood decoding of the system can be seen to be

$$
\arg \min _{s}\left\|\boldsymbol{x}-\sqrt{2 P}\left(\begin{array}{cc}
s_{1} & -\bar{s}_{2} \\
s_{2} & \bar{s}_{1}
\end{array}\right)\left[\begin{array}{l}
\sqrt{0.5 P} f_{1} g_{1} / \sqrt{\Omega P+1} \\
\sqrt{0.5 P} f_{2} g_{2} / \sqrt{\Omega P+1}
\end{array}\right]\right\|^{2}
$$

For the above ML decoder, the PEP, averaged over the channel coefficients, of mistaking the signal vector $s_{k}$ by $s_{l}$ has the following Chernoff bound based on (3) in [5]

$$
\begin{aligned}
& \mathrm{P}\left(\boldsymbol{s}_{k} \rightarrow \boldsymbol{s}_{l}\right) \leq \\
& E_{f_{i}, g_{i}}\left\{\exp \left(-\frac{\alpha P s_{k l}}{2\left(1+\alpha\|\boldsymbol{g}\|^{2}\right)}\left(\left|f_{1}\right|^{2}\left|g_{1}\right|^{2}+\left|f_{2}\right|^{2}\left|g_{2}\right|^{2}\right)\right)\right\}
\end{aligned}
$$

where $s_{k l}=\left\|s_{k}-s_{l}\right\|^{2}$ and $\alpha$ is a constant defined by $\alpha=0.5 P /(\Omega P+1)$. Since $\left|f_{i}\right|^{2} \sim \operatorname{Gamma}(\mathrm{m}, \Omega / m)$, $\left|g_{i}\right|^{2} \sim \operatorname{Gamma}(\mathrm{n}, \omega / n)$, by integrating over $f_{i}$, and using equation (3.326) in [6], we can obtain

$$
\begin{gathered}
\mathrm{P}\left(\boldsymbol{s}_{k} \rightarrow \boldsymbol{s}_{l}\right) \leq E_{g_{1}, g_{2}}\left\{h\left(s_{k l}, g_{1}, g_{2}\right)\right\} \\
=\frac{1}{(\omega / n)^{2 n}[\Gamma(n)]^{2}} \times \\
\quad \int_{0}^{\infty} \int_{0}^{\infty} h\left(s_{k l}, x, y\right)(x y)^{n-1} \exp \left\{-\frac{n}{\omega}(x+y)\right\} \mathrm{d} x \mathrm{~d} y
\end{gathered}
$$

where $h\left(s_{k l}, x, y\right)$ is defined by following equations

$$
h\left(s_{k l}, x, y\right)=\frac{1}{\left(s_{k l} \psi(x+y) x+1\right)^{m}} \frac{1}{\left(s_{k l} \psi(x+y) y+1\right)^{m}}
$$

with the function $\psi(\cdot)$ has the following expression

$$
\psi(z)=\frac{\Omega P}{2 m(2 \Omega+z)}
$$

In order to calculate the integration in (8), we apply the following approximation:

$$
\begin{aligned}
& \int_{0}^{\infty} \int_{0}^{\infty} h\left(s_{k l}, x, y\right)(x y)^{n-1} \exp \left\{-\frac{n}{\omega}(x+y)\right\} \mathrm{d} x \mathrm{~d} y \\
& \approx \int_{0}^{\infty} \int_{0}^{\infty} h^{\prime}\left(s_{k l}, x, y\right)(x y)^{n-1} \exp \left\{-\frac{n}{\omega}(x+y)\right\} \mathrm{d} x \mathrm{~d} y
\end{aligned}
$$

where $h^{\prime}\left(s_{k l}, x, y\right)$ is defined by following equation

$$
h^{\prime}\left(s_{k l}, x, y\right)=\frac{1}{\left(s_{k l} \psi(2 x) x+1\right)^{m}} \frac{1}{\left(s_{k l} \psi(2 y) y+1\right)^{m}}
$$

Let $\Omega P s_{k l} /(2 m)=k$, the right side of (11) will become

$$
I \triangleq\left[\int_{0}^{\infty} \frac{1}{\left(\frac{k x}{2 \Omega+2 x}+1\right)^{m}} x^{n-1} e^{-\frac{n}{w} x} \mathrm{~d} x\right]^{2} \triangleq T^{2}
$$

In order to calculate $I$, we divide the integral $T$ into three parts and approximate each of them as shown below

$$
\begin{aligned}
T & =\left(\int_{0}^{\Omega / k}+\int_{\Omega / k}^{3 \Omega / k}+\int_{3 \Omega / k}^{\infty}\right) \frac{1}{\left(\frac{k x}{2 \Omega+2 x}+1\right)^{m}} x^{n-1} \exp \left(-\frac{n}{w} x\right) \mathrm{d} x \\
& \cong \int_{0}^{\Omega / k} \frac{2^{m} \Omega^{m}}{\left(2 \Omega+\frac{\Omega}{2}\right)^{m}} x^{n-1} \exp \left(-\frac{n}{w} x\right) \mathrm{d} x
\end{aligned}
$$




$$
\begin{aligned}
& +\int_{\Omega / k}^{3 \Omega / k} \frac{2^{m} \Omega^{m}}{(2 \Omega+2 \Omega)^{m}} x^{n-1} \exp \left(-\frac{n}{w} x\right) \mathrm{d} x \\
& +\int_{3 \Omega / k}^{\infty} \frac{2^{m}(\Omega+x)^{m}}{(k x)^{m}} x^{n-1} \exp \left(-\frac{n}{w} x\right) \mathrm{d} x
\end{aligned}
$$

By applying equations (3.351.1), (3.351.2) and (1.111) in [6], we get the closed-form expression approximation of $T$ as follows

$$
\begin{gathered}
T \approx\left[\frac{4}{5}\right]^{m}\left[\frac{n}{\omega}\right]^{-n} \gamma\left(n, \frac{n \Omega}{k \omega}\right) \\
+\frac{1}{2^{m}}\left[\frac{n}{w}\right]^{-n}\left[\gamma\left(n, \frac{3 n \Omega}{k \omega}\right)-\gamma\left(n, \frac{n \Omega}{k \omega}\right)\right] \\
+\frac{2^{m}}{k^{m}} \sum_{i=0}^{m}\left(\begin{array}{c}
m \\
i
\end{array}\right)\left(\frac{n}{\omega}\right)^{-n+m-i} \Gamma\left(n-m+i, \frac{3 n \Omega}{k \omega}\right) \Omega^{m-i}
\end{gathered}
$$

where $\gamma$ and $\Gamma$ represent the incomplete gamma functions with the following definitions

$$
\gamma(\alpha, x)=\int_{0}^{x} e^{-t} t^{\alpha-1} \mathrm{~d} t, \quad \Gamma(\alpha, x)=\int_{x}^{\infty} e^{-t} t^{\alpha-1} \mathrm{~d} t
$$

So $T^{2}$ is the closed-form expression of the Chernoff bound approximation in (8).

\section{NUMERICAL RESUlTS AND SIMULATIONS}

In this section, we will show that the approximation of upper bound given in the section III is valid for various cases of the fading parameters $m, \Omega, n, \omega$ by numerical results.

The real value of the Chernoff bound is calculated by mathematical software. Apply Monte Carlo simulations with respect to channel variables to some cases of parameters, the numerical results (shown in Fig. 2) show that the approximation derived in section III is quite near to the real value. We take several case of parameters $(m, \Omega, n, \omega)$ as $(1,1,1,1),(1,2,1,3),(1,2,2,3),(2,1,3,1),(2,2,3,3),(2,3,3,5)$.

In the numerical results, without lose of generality, $s_{k l}$ is set to be 2, which is the case of BPSK modulation. In Fig. 2, the actual values of PEP at all parameter combinations of $(m, \Omega, n, \omega)$ are denoted by continuous lines, and the approximate values of PEP at all parameter combinations are denoted by single signs. Fig. 2 shows that the single signs are nearly coincided with the continuous lines, this is to say that the approximation given in Section III is very near to the actual value of PEP.

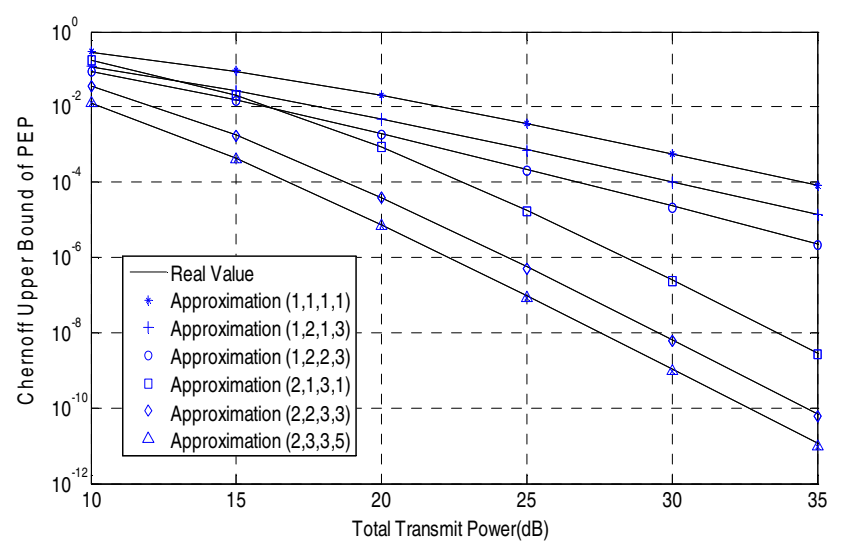

Figure 2 Real value and approximation of Chernoff upper bound

\section{CONCLUSION}

In this paper, an approximation of the Chernoff bound of the PEP for a Alamouti DSTC-relay system over Nakagami- $m$ fading channel is given, numerical results show the validity of the approximation.

\section{ACKNOWLEDGMENT}

This work is supported by National Science and Technology Key Special Projects (No. 2010ZX03003-002 \& 2010ZX03003-004), National Nature Science Foundation of China (No. 60972023), and Research Fund of National Mobile Communications Research Laboratory, Southeast University (No. 2011A06), and also supported by UK-China Science Bridge : R\&D of (B)4G Wireless Communications System.

\section{REFERENCES}

[1] J. N. Laneman, and G. W. Wornell, "Distributed space-time-coded protocols for exploiting cooperative diversity in wireless network," IEEE Trans. Inf. Theory, vol. 49, pp. 2415-2425, Oct. 2003.

[2] Y. Jing and B. Hassibi, "Distributed space-time coding in wireless relay networks," IEEE Trans. Wireless Commun., vol. 5, pp. 3524-3536, Dec. 2006.

[3] Y. Jing and H. Jafarkhani, "Distributed differential space-time coding in wireless relay networks," IEEE Trans. Wireless Commun., vol. 56, pp. 1092-1118, July 2008.

[4] B. Mahamy, A. Hjorungnes, and G. Abreuz, "Distributed GABBA space-time codes with complex signal constellations," Sensor Array and Multichannel Signal Processing Workshop, 5th IEEE, pp. 118-121, 2008.

[5] Y. Jing and H. Jafarkhani, "Relay power allocation in distributed spacetime coded networks with channel statistical information," IEEE Trans. Wireless Commun., vol. 10, pp.443-449, Feb. 2011.

[6] I. S. Gradshteyn and I. M. Ryzhik, Table of Integrals, Series and Products. Academic Press, 7nd ed., 2007. 